\title{
Controle Quântico Ótimo: Condições Necessárias de Optimalidade
}

\author{
Alexandre Coutinho Lisboa \\ Departamento de Engenharia de Telecomunicações e Controle, Escola Politécnica da Universidade \\ de São Paulo, EPUSP, São Paulo, SP,

\section{José Roberto Castilho Piqueira ${ }^{2}$} \\ Departamento de Engenharia de Telecomunicações e Controle, Escola Politécnica da Universidade \\ de São Paulo, EPUSP, São Paulo, SP.
}

\begin{abstract}
Resumo. Neste trabalho sobre Controle Quântico Ótimo, discutimos as condições básicas necessárias de optimalidade, a saber, o Princípio do Máximo de Pontryagin (PMP), tratado, inicialmente, do ponto de vista clássico, para sua posterior extensão para sistemas quânticos, no afã de estabelecer uma metodologia de busca de controles ótimos neste contexto físico.
\end{abstract}

Palavras-chave. Controle Quântico, Controle Ótimo, Mecânica Quântica, Informação Quântica.

\section{Introdução}

Em toda derivação de Condições Necessárias de Optimalidade, assume-se que o controle admissível $u *(t)$, obtido, é ótimo e substitui $u(t)$ com um controle ligeiramente diferente $u^{\varepsilon}$, no qual $\varepsilon$ é um parâmetro pequeno. O controle $u^{\varepsilon}$ é, ainda, admissível, e, do ponto de vista variacional, é chamado de uma variação de $u(t)$. Então, impõe-se que, para o funcional de custo $J, J=J(u)$, a seguinte desigualdade seja válida:

$$
J\left(u^{\varepsilon}\right)-J(u) \geq 0
$$

a qual dá as condições necessárias desejadas ao controle $u$. De acordo com o tipo de variação que se considera, diferentes condições de optimalidade são obtidas. Em primeira instância, podemos ter uma variação dita fraca de $u(t)$, a qual pode ser expressa em linguagem variacional como se segue:

\footnotetext{
1 alexandrecl@usp.br

2 piqueira@lac.usp.br
} 


$$
u^{\varepsilon}(t)=u(t)+\varepsilon v(t)
$$

em que, $\varepsilon$ é um parâmetro real, pequeno; e, $v(t)$, uma função contínua arbitrária, com as mesmas condições de contorno de $u(t)$.

As condições necessárias básicas de optimalidade ${ }^{3}$, em Controle Ótimo, são conhecidas como o Princípio do Máximo de Pontryagin (PMP), o qual pode ser encontrado em virtualmente todos os livros dedicados à teoria de Controle Ótimo, e, até mesmo, em alguns textos sobre Cálculo das Variações, como, por exemplo, em [2]. Neles, a abordagem do Princípio do Máximo de Pontryagin varia bastante em termos de apresentação, notação, detalhamento, sofisticação e rigor matemático empregados. Em [3], texto clássico e bastante empregado em cursos de Controle Ótimo, o PMP é apresentado de forma mais informal, de modo que o autor apresenta uma dedução heurística do Princípio. A referência original, geralmente citada na bibliografia de diversos autores, é o livro-texto, traduzido para o inglês, do próprio matemático soviético Lev S. Pontryagin e seus colaboradores [4], a qual se constitui em um tratamento extenso e rigoroso da Teoria de Otimização de Processos. Uma abordagem moderna, minuciosa e matematicamente rigorosa do PMP pode ser encontrada no livro-texto de Schättler e Ledzewicz [5], o qual apresenta o Princípio em seu Capítulo 2, para finalmente prová-lo de forma detalhada e rigorosa no Capítulo 4.

Tentaremos adaptar, na próxima Seção, as abordagens de [1] e de [5], tendo em vista que [1] presta-se melhor aos nossos propósitos, no tocante à aplicação do PMP para problemas de Controle Quântico Ótimo.

\section{O Princípio do Máximo de Pontryagin e Sua Extensão para Sistemas Quânticos}

Consideremos, agora, o caso mais geral, ou seja, em que se tem um Problema de Bolza, com um estado final livre, para um tempo final T. As condições do Princípio do Máximo de Pontryagin podem ser estabelecidas como se segue.

Seja um sistema de controle $\dot{x}=f(x, u)$ e um funcional de custo de Controle Ótimo, do tipo Bolza, definido como se segue:

$$
J(u) \equiv \phi(x(T), T)+\int_{0}^{T} L(x(t), u(t), t) d t
$$

no qual, as funções $\phi$ e $L$ correspondem, respectivamente, às funções de Mayer e de Lagrange, conforme apresentadas em nosso primeiro trabalho, e as assumiremos como sendo continuamente diferenciáveis.

\footnotetext{
3 Condições necessárias, porém, não suficientes, de modo que se pode encontrar um controle admissível $u^{*}$, que satisfaça as condições do PMP, em outras palavras, que seja um extremante das condições variacionais nele estabelecidas, mas que não seja o controle ótimo global $[3,5]$.
} 
Definamos, a seguir, o Hamiltoniano de Controle Ótimo, $h(t, \lambda, \lambda, x, u)$, $h: \mathfrak{R} \times \mathfrak{R}^{*} \times\left(\mathfrak{R}^{n}\right)^{*} \times \mathfrak{R}^{n} \times \mathfrak{R}^{n} \rightarrow \mathfrak{R}$, o qual denotamos, deliberadamente, com “ $h$ minúsculo", a fim de evitar potenciais confusões com o Operador Hamiltoniano Quântico, $\hat{H}$ :

$$
h\left(t, \lambda_{0}, \lambda, x, u\right) \equiv \lambda^{T} f(x, u)+\lambda_{0} L(x, u)
$$

em que, $f(x, u)$ é um campo vetorial suave; $x$, as variáveis de estado do sistema, de dimensão $n$; e $\lambda$ são os chamados co-estados do sistema, os quais são dados pelas seguintes equações adjuntas (conjunto de $n$ equações diferenciais ordinárias):

$$
\dot{\lambda}^{T}=-\lambda^{T} f_{x}-\lambda_{0} L_{x}
$$

para as quais devemos ter a condição de não-trivialidade dos multiplicadores, i.e.:

$$
\left(\lambda_{0}, \lambda(t)\right) \neq 0, \forall t \in\left[t_{0}, T\right]
$$

bem como, a condição de contorno terminal:

$$
\lambda^{T}(T)=-\phi_{x}(x(T))
$$

Estamos, agora, em condições de enunciar o seguinte:

Teorema 2.1. Princípio do Máximo de Pontryagin para um Problema de Bolza (com estado final livre).

Seja uma trajetória $x *(t)$ controlada que é solução do sistema $\dot{x}(t)=f(x(t), u(t))$, no intervalo $\left[t_{0}, T\right]$, com o controle $u(t)$ contínuo por partes. Se $u$ é ótimo $-u=u^{*}$ - então, existe um vetor $\lambda$, dito co-estado do sistema, o qual deve satisfazer as equações adjuntas (2.3), a condição de não-trivialidade (2.4) e a condição de contorno terminal (2.5). Ademais, o hamiltoniano de controle ótimo satisfaz, também, a seguinte desigualdade: $h\left(t, \lambda_{0}, \lambda(t), x^{*}(t), u^{*}(t)\right) \geq h\left(t, \lambda_{0}, \lambda(t), x(t), v(t)\right), \forall t \in\left[t_{0}, T\right]$, com os controles $u^{*}(t)$ e $v(t)$ pertencentes ao conjunto dos controles admissíveis $U$.

Passemos, doravante, a examinar as condições de optimalidade para problemas de Controle Quântico. Para os problemas de Controle Ótimo, no contexto físico de interesse para este artigo, ou seja, sistemas quânticos de dimensão finita (espectro discreto de autovalores), temos as seguintes expressões para um tal sistema e para o funcional de custo associado, conforme o que fora discutido em nosso trabalho anterior: 


$$
\begin{gathered}
\dot{x}=\tilde{H}(u) x \\
J(u)=\tilde{\phi}(x(T), T)+\int_{0}^{T} \tilde{L}(x(t), u(t), t) d t
\end{gathered}
$$

O Hamiltoniano de Controle Ótimo $h(\lambda, x, u)$ toma, então, a forma [1]:

$$
h(\lambda, x, u)=\lambda^{T} \tilde{H}(u) x-\tilde{L}(x, u)
$$

O controle ótimo $u$ tem que satisfazer $h(\lambda, x, u) \geq h(\lambda, x, v)$ com tal Hamiltoniano de Controle Ótimo. As equações adjuntas são:

$$
\dot{\lambda}^{T}=-\lambda^{T} \tilde{H}(u)+\tilde{L}_{x}
$$

as quais, desde que $\tilde{H}(u)$ seja anti-simétrico, podem ser escritas como [1]:

$$
\dot{\lambda}=\tilde{H}(u) \lambda+\tilde{L}_{x}^{T}
$$

com a condição terminal dada por $\lambda(T)=-\tilde{\phi}_{x}^{T}(x(T))$. Note que, caso $\tilde{L}_{x}=0$, isto é, o integrando do funcional de custo (2.7) não dependa do estado $x, x$ e $\lambda$ satisfazem a mesma equação diferencial, e as equações para $\lambda$ e $x$ são acopladas apenas através dos controles.

\section{Conclusões}

Procuramos expor os fundamentos da Teoria de Controle Quântico Ótimo, através da generalização da Teoria de Controle Ótimo Clássico para Sistemas Quânticos, de modo a apresentar o ferramental matemático básico, adequado para atacar problemas de controle ótimo em tal domínio físico. Com efeito, primeiramente, a partir de considerações oriundas da Dinâmica Quântica, expressamos as equações dinâmicas pertinentes e os funcionais de custo de controle ótimo quântico, em nosso primeiro artigo. Neste aqui, estendemos o Princípio do Máximo de Pontryagin para sistemas quânticos, de sorte a fornecer uma metodologia rigorosa para a obtenção das condições necessárias de optimalidade, a qual nos permite derivar controles ótimos para os mesmos. Ainda que a solução analítica de tais problemas de controle seja possível apenas para sistemas com baixa dimensionalidade e/ou dotados de simetrias que permitam simplificações, os fundamentos teóricos de Controle Quântico Ótimo embasam os métodos numéricos e computacionais, que são amplamente utilizados para resolver uma grande gama de problemas, quer de natureza prática ou 
tecnológica, quer de natureza teórica.

Esperamos que estes dois trabalhos possam estimular e fomentar pesquisas futuras, tanto teóricas, quanto aplicadas, uma vez que neles se procura fornecer o background teórico básico de Controle Quântico Ótimo, de forma sintética e rigorosa, e, não obstante, sem prejuízo na clareza da exposição.

\section{Referências}

[1] D. D'Alessandro, Introduction to Quantum Dynamics and Control. Boca Raton, FL: Chapman\&Hall / CRC, (2007).

[2] I. M. Gelfand, S. V. Fomin, S. V, Calculus of Variations. Mineola, NY: Dover Publications, (2000), (Reprint of the1963 Original Edition).

[3] D. E. Kirk, Optimal Control Theory: An Introduction. Englewood Cliffs, NJ: Prentice Hall, (1970).

[4] L. S. Pontryagin et al, The Mathematical Theory of Optimal Processes. New York: Interscience Publishers, Inc., (1962).

[5] H. Schättler, U. Ledzewicz, Geometric Optimal Control: Theory, Methods and Examples. New York: Springer Verlag, (Interdisciplinary Applied Mathematics), (2012). 